\title{
DESIGN AND PATH PLANNING SIMULATION OF PROBE ARM OF POWER GIS PIPELINE PROBE ROBOT
}

\author{
Xiao Liu ${ }^{1}$, Wei Wang ${ }^{1}$, Jianmin Wang ${ }^{1}$, Kai Wei ${ }^{1}$, Xiaohai Yin ${ }^{1}$ \\ ${ }^{1}$ Maintenance Branch of State Grid Shanxi Electric Power Company \\ Taiyuan, Shanxi 030006, China \\ Email: cgyliuxiao@sx.sgcc.com.cn
}

\begin{abstract}
With the improvement of science and technology, gas insulated metal closed switchgear (GIS) has been applied significantly in the power industry, and has become the core equipment in the substation. Once the fault occurs, it may cause serious economic losses and threaten the life safety of the staff. In addition, due to the special reasons in GIS, it is difficult for the traditional inspection method to achieve the non-dead corner inspection, and the risk of inspection operation is very high, which is easy to lead to gas poisoning and other problems. Based on this, according to the requirements of GIS pipeline detection, this paper designed and researched the detection arm of GIS pipeline detection robot, and carried out the simulation experiment of path planning. The results confirmed the feasibility of this design, which could effectively deal with the complex problems of internal results of GIS pipeline, effectively avoid obstacles and complete path planning. The purpose of this study is to promote the intelligent level of GIS pipeline inspection and maintenance operation, gradually replace the traditional inspection mode of power GIS, improve the inspection and maintenance efficiency and quality, and eliminate the occurrence of GIS faults from the root.
\end{abstract}

Keywords: GIS pipeline, Detection robot, Detection arm, Path planning, The simulation.

\section{Introduction}

In recent years, GIS pipeline has been widely used in power system due to its advantages of small footprint, compact structure and high reliability, and has become a key equipment in substation [1-4]. However, due to the fact that the GIS pipeline space is fully sealed, the inspection and maintenance work of GIS pipeline is inhibited. When a fault occurs, a large amount of time is needed for fault calibration and maintenance, which brings troubles to residents and commercial electricity consumption. In terms of the internal structure of GIS, the space is narrow, the maximum length of the compartment of $220 \mathrm{kV}$ main bus is $12 \mathrm{~m}$, and exhaust is needed after opening the cover, which increases the maintenance time of GIS.

After sealing the cover, it is necessary to carry out aeration, micro-water and leak detection experiments, so as to ensure the security of GIS equipment operation [5]. It can be seen that due to the internal spatial structure of GIS pipeline, the difficulty of inspection and maintenance is increased. In addition, the threat of toxic gas may exist inside the pipeline, which inhibits the manual inspection. Therefore, this paper will design and study the detection arm of the power GIS pipeline detection robot, aiming to replace the traditional operation mode of GIS pipeline through this research and improve the inspection efficiency and security of GIS pipeline.

\section{Structure Design and Verification of Detecting Arm of Power GIS Pipeline Detecting Robot}

\subsection{Configuration Design of Probe Arm}

GIS is composed of components such as pipes, disconnecting switches, circuit breakers, grounding switches, lightning arresters, mutual inductors, outgoing terminals and buses, and its internal structure is characterized by complexity [6]. According to the structural characteristics, the design of the probe arm should have the following characteristics: first, after the workspace of the probe arm is superimposed with the motion space of the vehicle body, the height of the probe arm should cover the probe space in the pipeline; Second, sufficient degree of freedom should be given to enable it to detect according to a specific trajectory. Third, it should have a sound control performance and stability. Therefore, the overall index design of the manipulator is as follows: the maximum expansion radius should not be less than $350 \mathrm{~mm}$; The mass of the body should not be greater than 1.5 $\mathrm{kg}$; The degree of freedom should not be less than 3 . 
Since the robot arm designed in this paper is mainly responsible for GIS pipeline detection without live operation, the visual range of the camera on the robot arm can be improved as much as possible. Three series joints close to the base were selected to determine the position of the detection mechanism. Whereinto, the axes of joint 2 and joint 3 are parallel to each other, and the position can be determined in a plane perpendicular to the axis. Joint 1 is perpendicular to joint 2 and joint 3 respectively. Under the action of its rotation, the position information of the end in Cartesian space can be determined. Under the action of these joints, the probe arm is given flexibility in space operation. The design of the probe arm mechanism is shown in Figure 1.

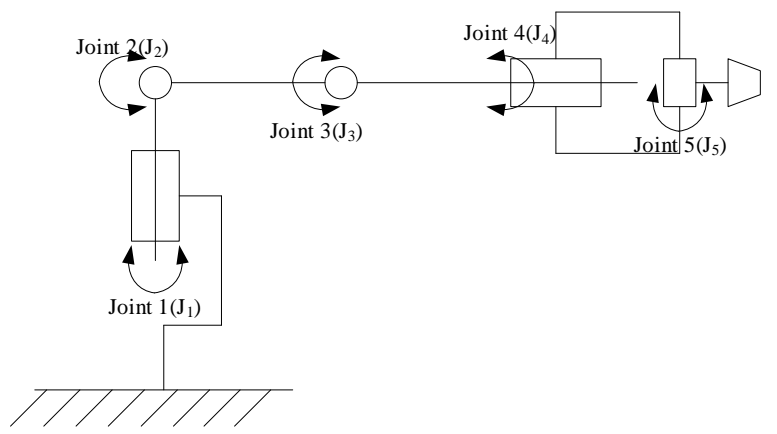

Figure 1: Schematic design of probe arm structure

It can be seen that the size of joint 2's big arm and joint 3's small arm are the key factors that determine the maximum space that can be reached at the end of the probe arm and its operability. After consideration, joint 2's big arm $L_{1}$ is set as $195 \mathrm{~mm}$, and joint 3's small $\operatorname{arm} L_{2}$ is set as $165 \mathrm{~mm}$. At this point, the structure and main parameters of the probe arm have been determined.

\subsection{Probe Arm Structure Design}

In the structural design of the probe arm, the design goal is to be lightweight, compact and reduce inertial force, that is, to reasonably arrange the direction and position of the force. According to the lightweight design goal of the probe arm, the arm connecting rod is selected as a hollow section. Among them, the hollow section can be divided into rectangular section and circular section, so it is necessary to complete the selection of the hollow section type through mechanical property analysis. The preliminary design is as follows: First of all, 6061 aluminum alloy is selected to complete the structural design of the forearm, so that it has good corrosion resistance and oxidation resistance effect. Then, the large arm structure is designed as far as possible without affecting the rotation range of the small arm, and its loading mass is $0.255 \mathrm{~kg}$. Secondly, the detection mechanism is connected with the forearm, but its detection flexibility is not enough to meet the detection of the dense parts of GIS pipelines. Therefore, a 2-DOF camera pan-head is set up at the end of the detection arm to assist the detection of the robotic arm to increase its detection flexibility. Due to the limitation of both mass and volume, the FS90R motor is chosen to control the two degrees of freedom at the end of the probe arm. Finally, the shoulder joint and the base need to bear a large force, but also need to bear a certain overturning moment. Among them, the base will be carried to the GIS pipeline robot body. During the movement of the GIS pipeline robot, the parts directly connected with it shall meet the design stiffness standard. After consideration, the mass of shoulder joint and base is $0.16 \mathrm{~kg}$ and $0.215 \mathrm{~kg}$, respectively. In addition, the thrust tapered roller bearing is installed in the base and the middle of the shoulder joint to share the corresponding axial and radial forces. After partial lightweight modification, the overall weight of the manipulator is $1.2 \mathrm{~kg}$. The overall structural design is shown in Fig.2.

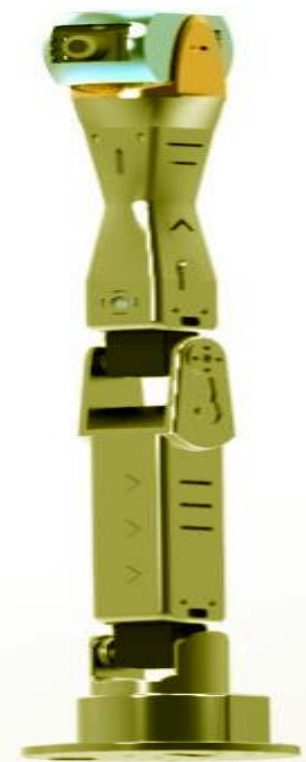

Figure 2: Assembly of the overall structure of the probe arm

\subsection{Lightweight Design of Probe Arm}

The purpose of the lightweight design of the detection ratio is to reduce the dimension of subsequent solution. For the design of the large arm structure, the most important factor is its overall mass, so the lightweight optimization of the detection ratio can be accomplished by quantifying its section size. The specific quantification process is as follows: First, the forearm is statically analyzed; Secondly, the forearm is meshed. Finally, the statics of the forearm are solved. After the above quantization process, the relevant parameters of the forearm are obtained as follows: the end mass is $0.104 \mathrm{~kg}$, the front torque is $0.063 \mathrm{~N} \cdot \mathrm{m}$, the force is $1.53 \mathrm{~N}$, and the average grid mass is 0.81 . In order to 
make full use of the material, the ANSYS Workbench topology optimization module was selected to optimize the forearm structure [7]. The optimized forearm section model is imported into ANSYS, and then under the same force and binding force, $60 \%$ of the section mass of the forearm is retained. Finally, it is imported into the modeling software for model modification, during which processing technology, positioning, installation and other factors need to be taken into account. The final modified forearm module is shown in Figure 3. At this time, the mass of the forearm is $0.111 \mathrm{~kg}$, the maximum stress is 1.257 $\mathrm{MPa}$, and the maximum deformation is $8.4^{*} 10^{-4} \mathrm{~mm}$.

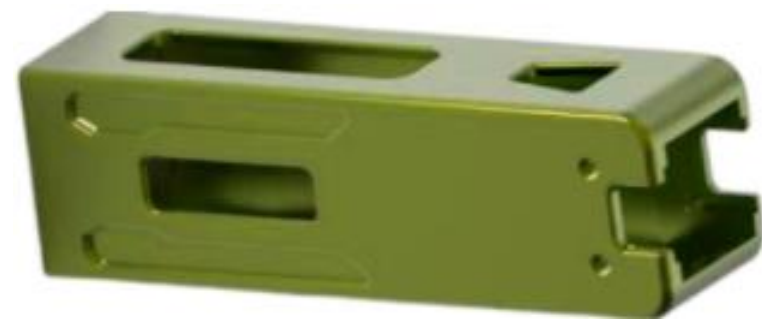

Figure 3: Modification results of the forearm module

\section{Kinematics Model Construction}

After the design of relevant parameters of the probe arm, the motion model of the probe arm was constructed and solved in order to better demonstrate the position and pose of each joint and the formation of assisted motion path planning. Specifically, the D-H coordinate system of the probe arm was constructed by using the D-H coordinate system rules, and then the manipulator was planned by using the Matlab simulation software to obtain the trajectory planning curve of polynomial difference of the probe arm, as shown in Figure 4.
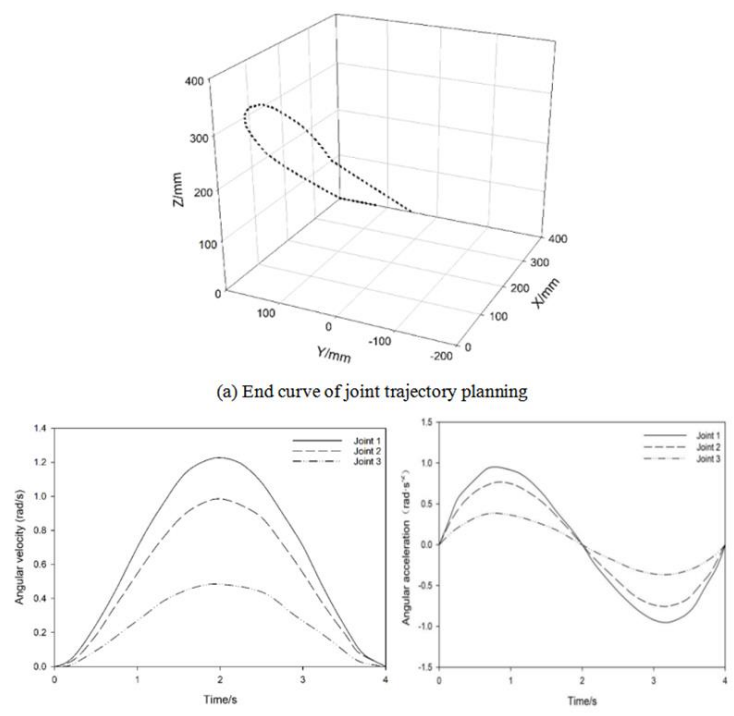

(b) Variation curve of joint angular velocity (c) Variation curve of joint angular acceleration

Figure 4: The trajectory planning curve of polynomial difference of the probe arm
As can be seen from Figure 4, the trajectory curve of the end of the probe arm is smooth, the angular velocity of the joint and its acceleration are stable, and there is no obvious abrupt change, indicating that the trajectory planning of the probe arm does not have any boundary crossing behavior and meets the requirements of path planning.

\section{Obstacle Avoidance Detection and Path Planning Simulation of Robot Probe Arm 4.1 Overview of Collision Detection Methods}

In the path planning of the probe arm, the most important thing is to check whether the probe arm can effectively avoid obstacles in the process of movement, that is, whether the probe arm will collide with GIS pipeline objects. Among them, collision detection can be divided into four ways: first, dynamic collision detection; Second, static collision detection; Third, image collision detection; Fourth, collision detection of objects. At the same time, there will be two situations in collision detection, one is whether the detection arm collides with the obstacles in the GIS pipeline; The second is to detect whether there is mutual interference behavior between the arm members. Nowadays, the common practice of collision detection is to present the obstacles in the object space and the detection arm itself in a three-dimensional way, and judge whether there is a collision by detecting whether these three-dimensional elements intersect. Spatial decomposition method and hierarchical bounding box method are two collision detection methods $[8,9]$ to detect whether there is intersection between three-dimensional elements. Among them, the main idea of spatial decomposition method is to divide the whole space into a number of (n) subspaces, and then regard three-dimensional objects such as obstacles and detection arms in the space as a number of regular subobject objects, and finally judge whether there is an intersection between subobjects in the same subspace. During the judgment, BSP tree method and octree method will be used. Of course, these methods are only suitable for monitoring the environment with few obstacles, while the detection efficiency in the complex environment has certain limitations. For the hierarchical bounding box method, the detection arm body and the obstacle can generate the bounding box, which needs to be generated in accordance with the top-down law. Specifically, the objects are surrounded by three-dimensional geometrical shapes. If there is no intersection between the bounding boxes, then it can be shown that the objects detected will not collide. Secondly, in order to avoid errors in detection, the intersecting bounding boxes in the first stage can be detected again, and the bounding boxes are continuously refined and iteratively detected. 
The degree of refinement determines the accuracy of detection results, that is, the higher the degree of refinement, the more accurate the detection results will be. In addition, the method of bounding generation is also very important, which is the key factor to determine the detection efficiency and accuracy. The types of bounding boxes commonly used include: directional bounding box (OOB bounding box), axis-to-it bounding box (AABB bounding box), discrete directed polyhedron, bounding ball, etc. For AABB bounding box, it is to realize the bounding object by a certain cube, and the three sides of the cube used are parallel to the benchmark coordinate axis respectively. By detecting whether there is intersection between cube and coordinate axis projection, we can judge whether there is collision problem between two objects.

This kind of bounding box has high efficiency in both generation and update, and does not take up too much memory space. As for the bounding box of the ball, it is the fastest one among these kinds of bounding boxes, which takes the center of the generated object as the center of the ball, and then expands the surrounding object to form the minimum sphere. The accuracy of the ball-bounding box can be determined by observing the plumpness of the geometry. The rotation of the ball-bounding object does not affect it, so there is no need to update the ball-bounding box when the object is rotating. In the face of poor isotropic geometry, the effect of the ball-enclosed box is more general. For the directional bounding box, the object can be surrounded by a cube, but it can only be generated effectively after the direction is fixed. When the direction of the object is changed, the direction of the directional bounding box will also change. In dynamic collision detection, real-time updates should be carried out according to requirements to ensure the accuracy of bounding box generation. However, the complexity of bounding box construction is related to the geometric complexity of the bounding object, and the higher the geometric complexity, the more complex the construction will be. For the discrete directed polyhedron, the envelopment surface has the characteristics of diversity, and is no longer limited to the cube. Meanwhile, the envelopment box has a relatively high precision, but the shortcomings are that the testing and updating methods are more complicated.

\subsection{Obstacle Avoidance Detection of Detection Arm}

Through the above analysis, we have a general understanding of the current collision detection methods and cleared up the advantages and disadvantages of various monitoring methods. In order to facilitate the later path planning of the probe arm, a cylinder is added to each rod of the probe arm to form a cylindrical bounding box, which starts from the waist joint and reaches the forearm. The diameter of the bounding box formed is $78 \mathrm{~mm}$, $56 \mathrm{~mm}$ and $74 \mathrm{~mm}$ respectively. The model of the bounding box of the probe arm is shown in Figure 5 .

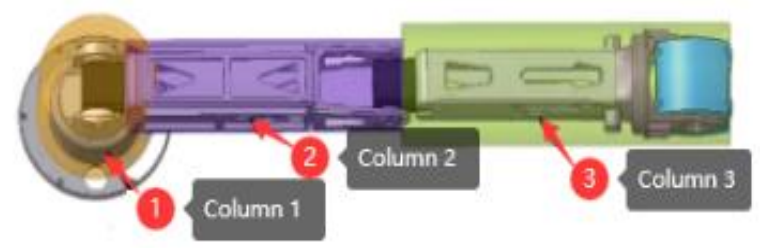

Figure 5: Bounding box model of probe arm

Through the analysis of the internal structure of GIS pipeline, it is found that there are some barriers such as insulators and connectors. In order to reduce the difficulty of detecting the obstacle avoidance of the detection arm, the ball-encasing box is used to wrap it. When detecting the obstacle avoidance of the detection arm, the radius $R$ of the cylinder can be superimposed on the enveloping ball $r_{2}$, so that the cylinder can be simplified into A line segment, and the radius of the enveloping ball is $R=r_{1}+r_{2}$. In each obstacle avoidance test, the coordinate value of the joint can be obtained by using the forward kinematics equation according to the known joint Angle, which is the coordinate of the end point of the line segment. If the two endpoints of the simplified line segment are A and B, and the spherical center is $\mathrm{C}$, then there are three situations encountered in obstacle avoidance detection: First, when $|\overrightarrow{A C}|-R<0$, or $|\overrightarrow{B C}|-R<0$, then some of the end points of the line segment will be inside the surrounding ball, and the probe arm will collide with the obstacle. Second, if $|\overrightarrow{A C}|-R>0$ and $|\overrightarrow{B C}|-R>0$ then the two endpoints of the line segment are not in the surrounding ball. In this case, the required distance between the center of the ball and the line segment is calculated by the formula as shown in Equation 1.

$$
\mathrm{d}=\frac{|\overrightarrow{A B} \times \overrightarrow{A C}|}{|\overrightarrow{A B}|}
$$

When $d>R$, the probe arm will not collide with obstacles inside the GIS pipeline. If $d<R$, then the third situation needs to be entered, and further judgment can only be made after the analysis of $\angle \mathrm{CAB}$ and $\angle \mathrm{CBA}$,As shown in Equation 2.

$$
\angle \mathrm{CAB}=\frac{\overline{\mathrm{AC} \cdot} \cdot \overline{\mathrm{AB}}}{|\overline{\mathrm{AC}}||\overline{\mathrm{AB}}|}, \angle \mathrm{CBA}=\frac{\overline{\mathrm{BC}} \cdot \overline{\mathrm{BA}}}{|\overline{\mathrm{BC}}||\overline{\mathrm{BA}}|}
$$

If both angles are less than $90^{\circ}$, it means that the line segment intersects with the bounding ball, and the detecting arm will collide with the obstacles inside the GIS pipeline; otherwise, obstacles can be effectively avoided. 


\subsection{Simulation Experiment of Probe Arm Path Planning}

On the basis of joint space, RRT-Connect path planning algorithm is used to carry out a simulation experiment on the path planning of the probe arm [10]. Assuming that there is an obstacle in the GIS pipeline, the radius is $0.1 \mathrm{~m}$ after being processed by the bounding box, and the position in the base coordinate system is $((0.1 m, 0.2 m, 0.2 m)$. Using the $\mathrm{D}-\mathrm{H}$ coordinate system, the initial state of the probe arm can be obtained as $\mathrm{q}_{\text {start }}=\left(0^{\circ}, 90^{\circ}, 0^{\circ}, 0^{\circ}, 0^{\circ}\right)$. After setting the target position without collision, the inverse kinematics equation is used to calculate the Angle of the target attitude joint, which is $q_{\text {goal }}=\left(110^{\circ}, 122^{\circ}, 53^{\circ}, 134^{\circ}, 65^{\circ}\right)$.
At this point, let the initial step $p=0.95$, then the comparison probability is step $=0.1$ and the maximum number of cycles $\mathrm{N}=2200$. After setting and solving relevant parameters, the path planning of the probe arm can be carried out. In the process of path planning, it may face the way of planning failure, which is the characteristic of its randomness.

In this paper, 10 simulation experiments on the path planning of the probe arm were all successful, and the average number of cycles was 75.1.

Figure 6 shows the extension tree generated after successful path planning. Then, the starting point and the index of the parent node are used to obtain the effective detection path points of the probe arm, and the final planned path is shown in Figure 7.

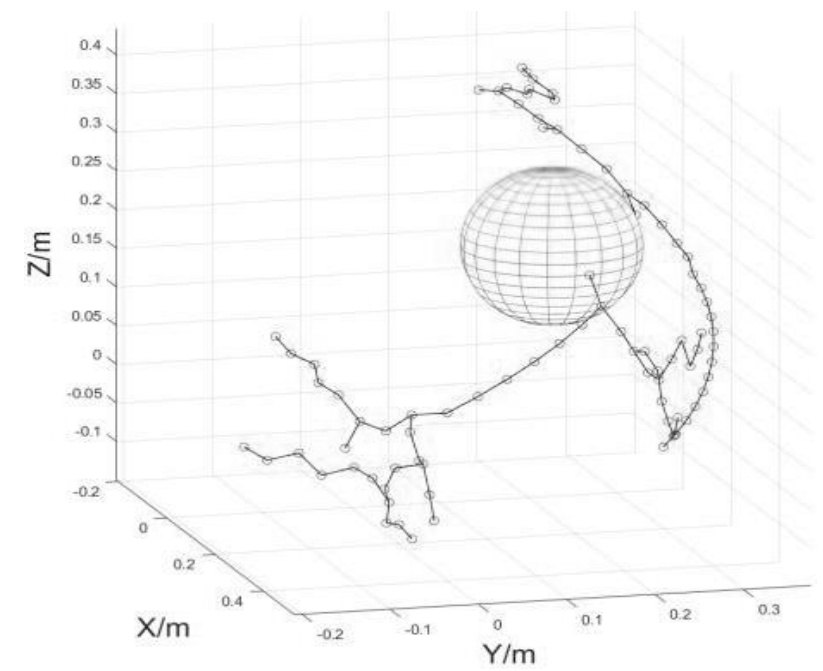

Figure 6: Expansion tree after path planning generation

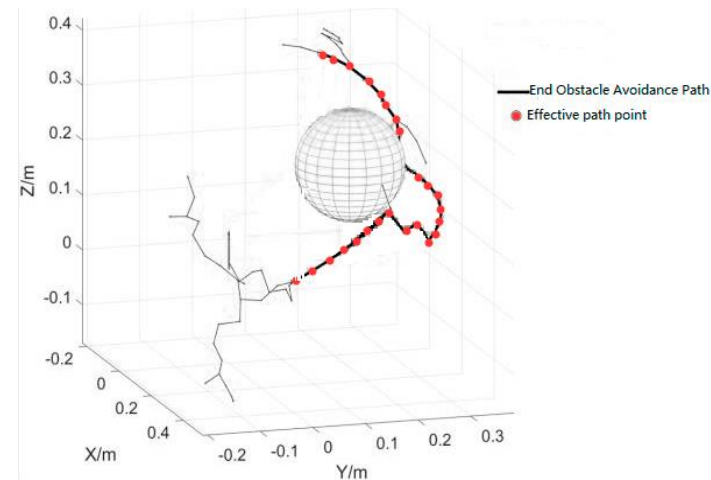

Figure 7: The valid probe path obtained by searching the parent node

As long as the probe arm detects the GIS pipeline according to this route, it can effectively avoid obstacles. At this point, if the step size is adjusted to 0.3 , after 10 simulations, the average number of cycles is 26.5 . It can be seen that with the increase of step size, the path planning speed and path irsmoothness of the probe arm will accelerate and worsen, as shown in Figure 8.

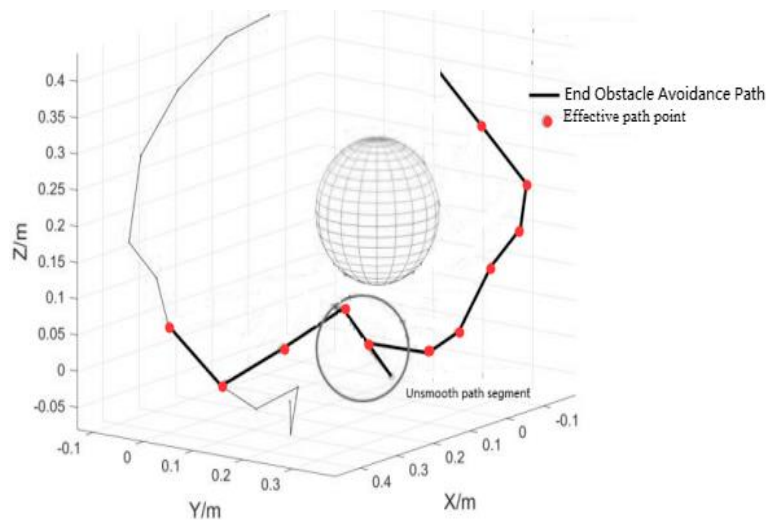

Figure 8: When the step size is 0.3, the probe arm plans the path

It can be seen from the simulation experiment that the probe arm can effectively deal with the obstacles in the GIS pipeline and complete the collision free path planning under the RRT-Conneect path planning algorithm. 


\section{Probe Arm Testing}

According to the structure design of the probe arm, the physical production of the probe arm was completed, and the preliminary prototype was obtained, as shown in Figure 9. Futaba extension line is used as the rudder pole machine line of the camera detection machine, and the connection between the bus steering gear is completed by using the puncture type $2 \mathrm{~mm}$ segment line. The steering gear control interface, PWM steering gear interface, external resource interface and so on are provided. At the same time, a variety of sensors such as infrared can be extended. After the prototype design is completed, it is necessary to control and debug the steering gear of the detection mechanism at the end of the detection arm. Among them, the steering gear has two forms: the first is the Angle control steering gear; The second is the $360^{\circ}$ speed control steering gear. In order to realize the reasonable control of the rotation speed and Angle of the detection mechanism at the end of the detection arm, two kinds of steering gear are selected for dismantling.

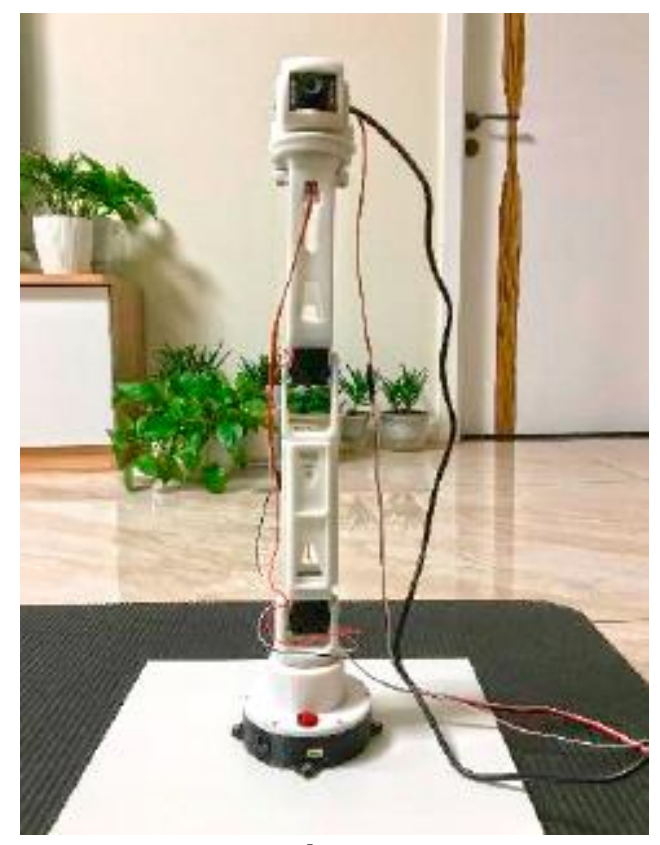

Figure 9: Probe arm prototype

In order to ensure the feasibility of the detection effect of the detection arm, the imaging effect of the camera is analyzed. Because the camera designed in this paper has the characteristics of wide dynamics and carries four infrared night vision LED lights, it can effectively deal with the problem of low illumination environment in GIS pipeline. In the lowillumination environment, the detection effect of the detection arm camera is shown in Figure 10.

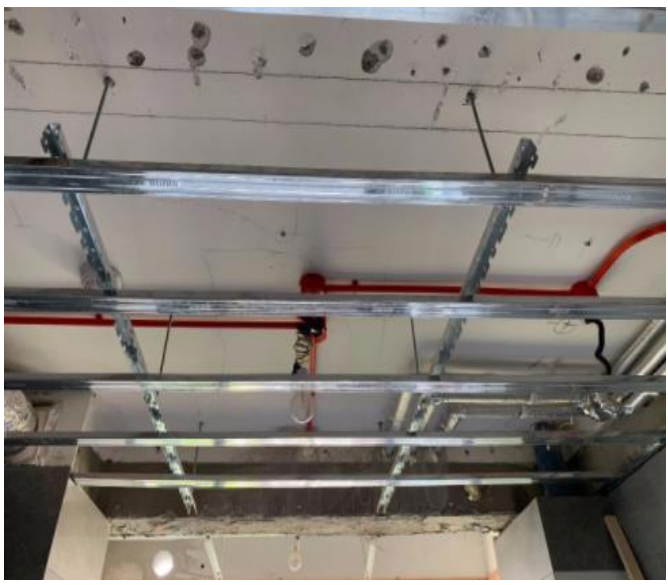

(a) Imaging by probe arm camera

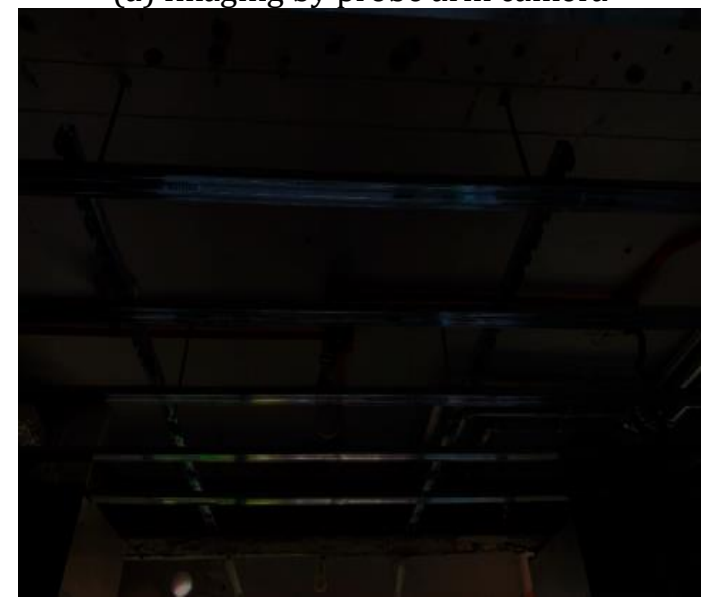

(b) The physical environment

Figure 10: Detection effect of detector arm camera

In the case of weak light, the robot camera can still carry out clear detection, and the comparison of images has no distortion. The detection effect is relatively good, which is conducive to the GIS pipeline defect location or object feature calibration work. After the camera detection of the detection arm was finished, the operation of the motor of the detection arm was detected. It was found that, combined with the inversion pulse time of the joint at the end of the detection arm, the camera could carry out all-round detection of the GIS pipeline from a $60^{\circ}$ perspective. At the same time, there is no vibration problem of the probe arm, which can realize the change of specific joint Angle. The operation of the manipulator arm is relatively stable, and the working performance of each joint is better, so it can be put into practice to complete the detection work of GIS pipeline.

\section{Conclusions}

To sum up, this paper first carries out the configuration design, structural design and lightweight design of the GIS pipeline detection arm, and carries out the corresponding check processing, and gives the overall assembly of the detection arm. 
Then, the kinematics model of the probe arm is constructed, the D-H coordinate system of the probe arm is given, and the related parameters are calibrated. At the same time, the trajectory planning of quintic polynomial difference based on joint space is carried out, and the results show that the design is reasonable. Secondly, RRT-Connect algorithm is used to carry out the path planning simulation experiment on the GIS pipeline probe arm.

The results confirm the fact that the GIS pipeline probe arm designed in this paper can carry out effective path planning and effective obstacle avoidance. Finally, the shooting effect of the detector arm was verified by experiments. According to the test results, the detector arm can effectively deal with the interference of low illumination environment and obtain good shooting effect. At the same time, the motion performance and pose are very friendly, which can meet the needs of GIS pipeline internal complex space inspection, efficient inspection and calibration.

\section{Acknowledgement}

This study was supported by State Grid Shanxi Electric Power Company Science and Technology Project: Research and application of key technologies for intelligent detection of suspended dense GIS Equipment Group in UHV Substation (Project fund No.: 52051020008A).

\section{References}

[1] Liang GL, Cui YF, Wang ZQ. "Analysis and Treatment of Air Leakage Causes of $330 \mathrm{kV}$ GIS Pipeline Bus," Power Safety Technology, 2018, 20(10):33-35.
[2] Luo HJ, Chen XT, Zhang J, Wu ZY, Zhao ZF, Li ZG. "Research on Finite Element Structure Analysis of GIS Equipment," Zhejiang Electric Power, 2019, 38(12):99-106.

[3] Tang FQ, Liu RH, Yang XH, Dong ZC, Geng LZ. "Structural Parameter Design and Motion Analysis of GIS Pipeline Inspection Robot," Journal of Electric Power, 2017, 32(05):397-403.

[4] Cao S Y. "Circuit Modeling of L-shaped Corner, Stand and Pan-type Insulator in GIS Pipeline," North China Electric Power University (Beijing), 2017.

[5] Zhang SL, He YS, Li NY, Peng ZR. "Finite Element Simulation Analysis of SF_6 Gas Insulated Composite Tube Structure for $1100 \mathrm{kV}$ GIS," Insulation Materials, 2019, 52(04):50-56.

[6] Hao XD. "Development and Development of a 363 kV Gas Insulated Metal Closed Switchgear," Electric Times, 2018(04):91-94.

[7] Liu ZH, Yang YF, Tian SL, Dai ZR. "Static Analysis of 6-DOF Manipulator Based on ANSYS," Coal Mine Machinery, 2020, 41(12):75-77.

[8] Yan A. "The spatial-temporal evolution of regional economic disparities in China," Statistics and Decision, 2020, 36(20):86-90.

[9] Geng H, Gao LL. "An Improved Hybrid Hierarchical Bounding Box Collision Detection Algorithm for Aircraft Virtual Maintenance," Science Technology and Engineering, 2018, 18(21):63-68.

[10] Sun LS. “Obstacle-avoidance Path Planning of Welding Robot Based on Improved RRT-Connect Algorithm," Automation \& Instrumentation, 201, 36(05):47-52. 